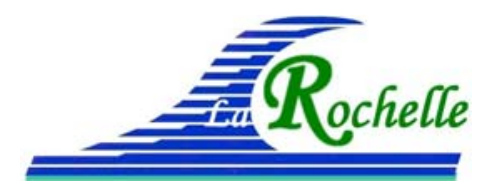

XVèmes Journées Nationales Génie Côtier - Génie Civil

La Rochelle, 29 au 31 mai 2018

DOI:10.5150/jngcgc.2018.095 ～(C) Editions Paralia CFL

disponible en ligne - http://www.paralia.fr - available online

\title{
Erodimètre triaxial de grandes dimensions pour la caractérisation de la sensibilité à l'érosion et du comportement mécanique de sols grossiers
}

\section{Didier MAROT ${ }^{\mathbf{1}}$, Fateh BENDAHMANE, Rinah ANDRIANATREHINA ${ }^{\mathbf{1}}$, Rachel GELET ${ }^{1}$}

1. Université de Nantes, Institut GeM, UMR CNRS 6183, 58 rue Michel Ange, SaintNazaire, France.

didier.marot@univ-nantes.fr

\section{Résumé :}

Les phénomènes d'érosion interne et d'érosion externe sont les principales causes des instabilités que peuvent subir les ouvrages hydrauliques en terre. La suffusion est l'un des quatre mécanismes d'érosion interne et mobilise les particules fines du sol qui peuvent se déplacer entre les grains de la fraction grossière. Dans la littérature, les conséquences de la suffusion sur le comportement mécanique des sols ont déjà fait l'objet de plusieurs travaux de recherche. Toutefois, les conclusions des travaux susmentionnés apparaissent controversées voire opposées. Les objectifs de cette étude sont donc de caractériser les modifications du comportement mécanique induites par le développement de la suffusion. Un érodimètre triaxial de grandes dimensions a été développé afin de réaliser des essais sur des sols grossiers fréquemment rencontrés dans les digues de France. L'analyse de la sensibilité des sols à la suffusion est menée à l'aide d'une méthode d'interprétation basée sur l'énergie dissipée par le fluide interstitiel et la masse érodée cumulée. Les résultats des essais de suffusion et de résistance mécanique montrent l'effet significatif du développement de la suffusion sur la résistance mécanique des sols testés. Dans le cas des sols testés, la suffusion complète induit une diminution de la contrainte déviatorique au pic.

Mots-clés :

Digue, Barrage, Suffusion, Erodimètre, Energie d'écoulement, Résistance mécanique.

\section{Introduction}

La suffusion est un processus d'érosion qui mobilise, sous l'action d'un écoulement interne, les particules fines des sols qui se déplacent dans les vides interstitiels de la fraction grossière. Ce phénomène est complexe car il est le résultat du couplage de trois processus : le détachement, le transport et la possible filtration de particules fines. Ces processus couplés peuvent produire une modification de la distribution granulométrique, de la porosité et donc du comportement mécanique du sol et de sa conductivité hydraulique. 


\section{Thème 7 - Risques côtiers}

FELL et FRY (2013) ont identifié trois critères qui doivent être satisfaits pour que la suffusion se produise : un critère géométrique, un critère de chargement mécanique et un critère hydraulique. 1) Le critère géométrique précise que la taille des particules fines doit être plus faible que la taille des vides entre les particules grossières. 2) Les particules fines ne doivent pas remplir entièrement les vides interstitiels afin que ces particules ne contribuent pas à supporter la contrainte effective. 3) Les auteurs considèrent que la vitesse de l'écoulement doit être suffisante pour entraîner les particules fines au travers du squelette granulaire.

La distribution granulométrique des grains constituant le sol a une influence déterminante sur les deux premiers critères. De ce fait, de nombreuses analyses de la distribution granulométrique ont été menées afin d'identifier la sensibilité potentielle d'un sol à la suffusion (notamment KENNEY \& LAU, 1985 ; WAN \& FELL, 2008 ; CHANG \& ZHANG, 2013a). Le troisième critère caractérise l'action de l'écoulement du fluide interstitiel qui entraîne le détachement puis le transport des particules fines. SKEMPTON et BROGAN (1994) ont proposé de définir l'initiation de la suffusion par l'accroissement de la conductivité hydraulique et de caractériser le chargement hydraulique correspondant par le gradient hydraulique, dénommé gradient hydraulique critique. Cependant, la filtration de certaines particules préalablement détachées peut induire un colmatage du milieu poreux et ainsi entraîner une diminution de la conductivité hydraulique (REDDI et al., 2000 ; BENDAHMANE et al., 2008). En conséquence, MAROT et al. (2016) prennent en compte les variations simultanées de la charge hydraulique et du débit pour caractériser le chargement hydraulique. Ils expriment la puissance dissipée par l'écoulement sous la forme :

$$
P_{\text {flow }}=Q \gamma_{\mathrm{w}} \Delta h
$$

où : $Q$ est le débit volumique [exprimé en $\mathrm{m}^{3} \cdot \mathrm{s}^{-1}$ ], $\gamma_{\mathrm{w}}$ le poids volumique de l'eau [N.m${ }^{3}$ ] et $\Delta h$ la perte de charge hydraulique [m].

MAROT et al. (2011) ont exprimé l'indice de résistance à l'érosion par :

$$
I_{\alpha}=-\log \left(\frac{m_{\text {dry }}}{E_{\text {flow }}}\right)
$$

où Eflow est l'énergie dissipée par l'écoulement [J], qui est déterminée par intégration temporelle de la puissance instantanée et $m_{d r y}$ est la masse érodée cumulée $[\mathrm{kg}]$. Cet indice est déterminé à la fin du processus de suffusion, c.à.d. lors d'une décroissance du taux d'érosion et de la stagnation de la conductivité hydraulique. A partir de cette approche énergétique et grâce à 23 essais menés avec un érodimètre triaxial, six catégories de sensibilité à la suffusion ont été proposées : de très érodable à très résistant (MAROT et al., 2016). De précédentes études ont montré que cette approche énergétique permet d'évaluer avec précision la sensibilité à la suffusion, sous différents historiques de chargement hydraulique (ROCHIM et al., 2017) et pour différentes tailles d'échantillons (ZHONG et al., 2018). 


\section{XVèmes Journées Nationales Génie Côtier - Génie Civil \\ La Rochelle, 29 au 31 mai 2018}

Cependant, les conséquences de la suffusion sur le comportement mécanique du sol constituent toujours une question ouverte et les conclusions de plusieurs études présentes dans la littérature apparaissent contradictoires. A l'aide d'une cellule triaxiale modifiée, CHANG et ZHANG (2013b) ont mené des essais de compression, suivant différents états de contraintes, sur un sol pulvérulent à distribution lacunaire. Ils concluent qu'après une perte significative de particules fines, le comportement (en conditions drainées) initialement dilatant devient contractant et que le déviateur maximal diminue. De la même manière, KE et TAKAHASHI (2015) ont testé trois mélanges lacunaires composés de deux sables et ils ont montré que la résistance au cisaillement diminue après la suffusion. Peuvent aussi être mentionnées les modélisations en éléments discrets réalisées par SCHOLTES et al. (2010) qui indiquent que la résistance au cisaillement décroît quand des particules fines sont érodées. A l'inverse, STERPI (2003) a testé des échantillons bien gradués de sable silteux et concluait que la perte partielle ou totale de particules fines induit un accroissement de la raideur et de la résistance au cisaillement. Cependant il importe de souligner que ces essais triaxiaux drainés ont été menés sur des échantillons non érodés, c'est-à-dire sur des échantillons homogènes qui ont été reconstitués avec un pourcentage de particules susceptible de représenter un échantillon après suffusion. Les conclusions de ces recherches peuvent être contradictoires à cause de la nature de la distribution granulométrique : discontinue pour les premières études mentionnées et bien graduée dans le dernier cas. Mais une autre raison peut provenir des hétérogénéités induites par la suffusion qui ne peuvent être reproduites par un échantillon reconstitué, même si la distribution globale de l'échantillon érodée est respectée.

\section{Dispositif expérimental et matériaux testés}

\subsection{Caractéristiques principales du prototype}

Un dispositif triaxial spécifique a été développé afin d'étudier les conséquences de la suffusion sur le comportement mécanique du sol. Cet appareillage permet de contrôler indépendamment le chargement hydraulique et le chargement mécanique (cf. Figure 1). Par ailleurs cet appareillage est doté d'une cellule triaxiale qui permet de tester des échantillons de $200 \mathrm{~mm}$ de diamètre et de $500 \mathrm{~mm}$ de haut. Cette spécificité autorise la réalisation d'essais sur des matériaux grossiers qui sont fréquemment rencontrés dans les digues en France et qui évite un écrêtage de la fraction grossière dont l'influence sur les propriétés hydromécaniques des sols est mal connue.

Pour le chargement hydraulique, le chapeau posé sur la surface supérieure de l'échantillon comprend un lit de gravier afin d'assurer une diffusion uniforme du fluide injecté. L'embase de la cellule est en forme d'entonnoir pour éviter tout dépôt et colmatage. La conduite de récolte de l'effluent est branchée à une boite de récolte qui contient un dispositif rotatif avec 8 béchers, afin de collecter les particules érodées au 


\section{Thème 7 - Risques côtiers}

cours du temps. Pour l'ensemble des essais réalisés dans le cadre de cette étude, les échantillons sont placés sur un tamis d'ouverture 1,2 mm, lui-même fixé sur une grille support d'ouverture $10 \mathrm{~mm}$. Un des deux débitmètres électromagnétiques que comprend le dispositif est sélectionné en fonction de la gamme de débit du fluide injecté. Le circuit hydraulique d'alimentation est constitué de 2 réservoirs sous pression d'une contenance de $200 \mathrm{~L}$ chacun, qui sont utilisés alternativement pour assurer la continuité du chargement hydraulique. La différence de pression interstitielle est mesurée à l'aide d'un capteur de pression branché à deux prises de pression, l'une au niveau du chapeau supérieur et la seconde au niveau de l'entonnoir de l'embase.

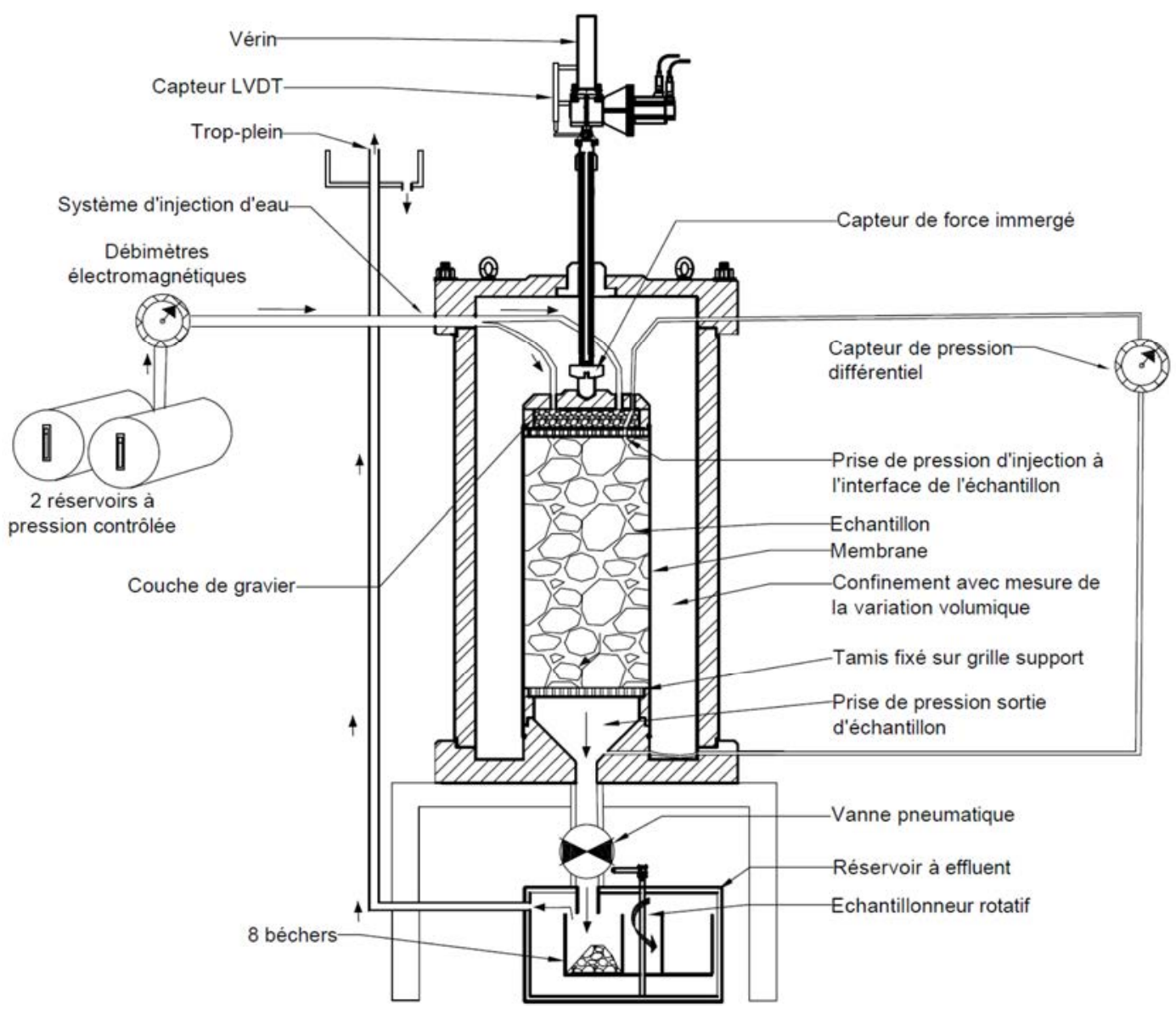

Figure 1. Représentation schématique de l'érodimètre triaxial de grandes dimensions

Un vérin électrique et un bâti support composent le système de chargement mécanique. Un capteur immergé permet la mesure de la force axiale appliquée. Le déplacement vertical du piston et donc la déformation axiale de l'échantillon sont déterminés à l'aide d'un capteur Linear Variable Differential Transducer (LVDT). Enfin, la pression de confinement est générée à l'aide d'un réservoir sous pression. Au cours d'un essai de résistance mécanique, la déformation volumique de l'échantillon est déterminée par 


\section{XVèmes Journées Nationales Génie Côtier - Génie Civil La Rochelle, 29 au 31 mai 2018}

mesure de la variation de volume d'eau interstitielle. Par contre, au cours d'un essai de suffusion, cette mesure n'étant pas possible à cause de l'écoulement, la déformation volumique est déterminée à partir de la variation de volume d'eau de confinement et en considérant les déplacements éventuels du vérin. Le pilotage du dispositif et l'acquisition des données sont réalisés à l'aide d'une centrale et d'un code écrit par les auteurs sous Labview.

\subsection{Matériaux testés}

La sensibilité à la suffusion et ses conséquences sur le comportement mécanique ont été évaluées pour trois échantillons de sols grossiers bien gradués (échantillons nommés 5.2-5.7, 6.2-6.7 et 11.2-11.7) provenant d'une même digue de France. Le nom des échantillons est relatif à la profondeur à laquelle ils ont été prélevés dans l'ouvrage. Un granulomètre laser a été utilisé afin de mesurer la distribution granulométrique de ces trois échantillons (cf. Figure 2) avec de l'eau déminéralisée mais sans agent défloculant afin d'être dans les mêmes conditions que lors d'un essai de suffusion.

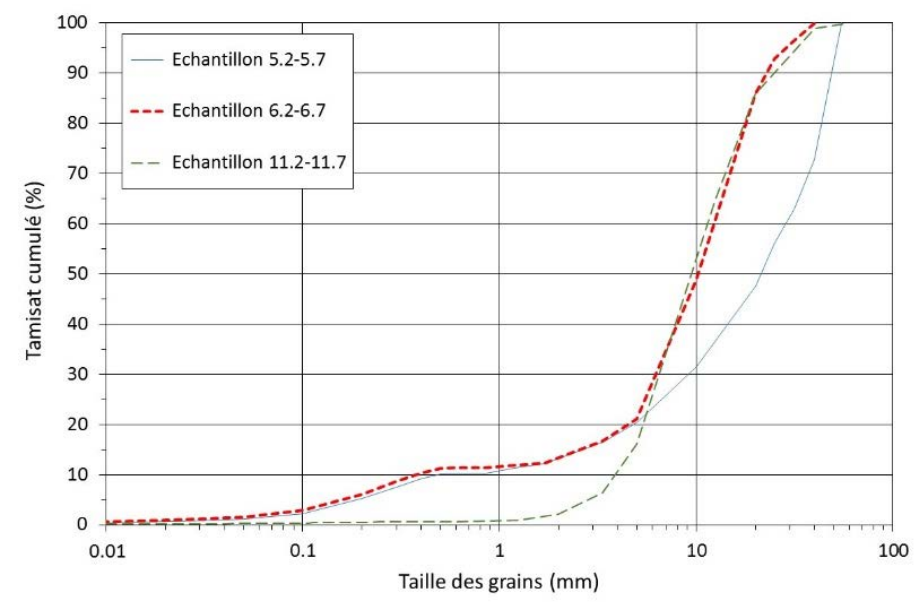

Figure 2. Distribution granulométrique des trois échantillons testés

Selon le critère granulométrique proposé par Kenney et Lau, les échantillons 5.2-5.7 et 6.2-6.7 sont instables. Cependant, le critère de Chang et Zhang abouti à la conclusion opposée. Selon les critères de Kenney et Lau, de Chang et Zhang et de Wan et Fell, l'échantillon 11.2-11.7 est classé stable.

\subsection{Programme expérimental}

La saturation et la consolidation sont réalisées conformément à la procédure décrite par BENDAHMANE et al. (2008). Pour chaque échantillon, le programme comprend deux essais : un premier essai de compression triaxiale monotone est réalisé afin de déterminer la résistance du sol intact en conditions drainées. L'objectif du second essai 


\section{Thème 7 - Risques côtiers}

est d'évaluer la résistance mécanique du sol après suffusion et s'effectue en 3 étapes successives :

i) Le chemin de chargement mécanique appliqué est identique à celui appliqué à l'échantillon intact, jusqu'à ce que la valeur de déviateur atteigne la contrainte géostatique appliquée à l'échantillon in-situ.

ii) Sous cet état de contrainte, un essai de suffusion est mené à l'aide d'un écoulement vertical descendant sous gradient hydraulique croissant par paliers.

iii)Au cours de la troisième étape, la contrainte déviatorique est augmentée jusqu'à la rupture de l'échantillon.

\section{Résultats et discussion}

\subsection{Conductivité hydraulique et taux d'érosion}

La figure 3(a) présente les valeurs du gradient hydraulique appliqué au cours des essais de suffusion et la figure 3(b) présente l'évolution correspondante de la conductivité hydraulique.

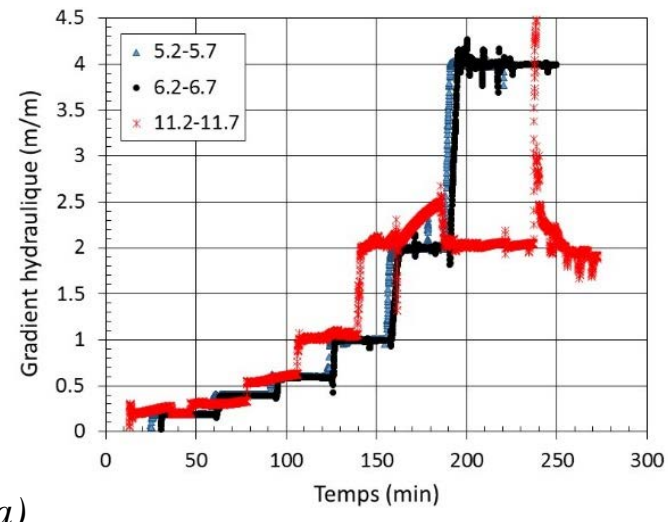

(a)

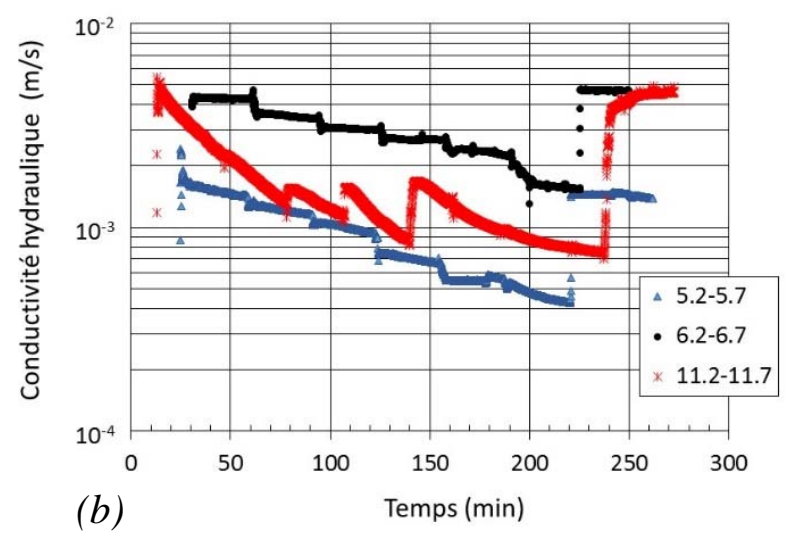

(b)

Figure 3. Evolutions au cours des essais de suffusion de (a) le gradient hydraulique appliqué, (b) la conductivité hydraulique

Le taux d'érosion moyen est également déterminé par l'équation (3) et son évolution au cours du temps est présentée par la figure 4.

$\dot{\mathrm{m}}=\frac{m(\Delta \mathrm{t})}{\mathrm{S} \Delta \mathrm{t}}$

avec $m$ la masse sèche érodée pendant la durée $\Delta t$ et $S$, la section transversale de l'échantillon. 


\section{XVèmes Journées Nationales Génie Côtier - Génie Civil \\ La Rochelle, 29 au 31 mai 2018}

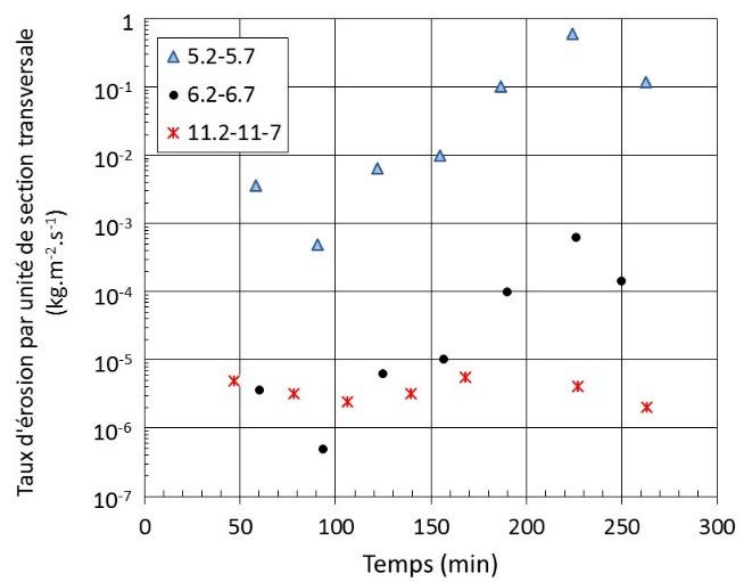

Figure 4. Evolutions au cours des essais de suffusion du taux d'érosion moyen.

Pour chacun des échantillons, globalement la conductivité hydraulique diminue. Cependant au début de l'application de chaque palier de gradient hydraulique, il convient de noter le fort accroissement de la conductivité hydraulique. Ce soudain accroissement est immédiatement suivi par une lente diminution de la conductivité. Lorsque le gradient hydraulique atteint la valeur de 4, la brusque augmentation de la conductivité est aussi mesurée mais simultanément un fort taux d'érosion est aussi mesuré. Ces augmentations simultanées suggèrent qu'un colmatage limitait l'écoulement mais qu'il pouvait être détruit par le brusque accroissement du chargement hydraulique. Finalement, la conductivité hydraulique tend à se stabiliser et le taux d'érosion décroît.

\subsection{Caractérisation de la sensibilité à la suffusion}

La sensibilité à la suffusion est déterminée à l'aide de l'approche énergétique : le chargement hydraulique est représenté par l'énergie dissipée par le fluide interstitiel qui est calculée jusqu'à la stabilisation de la conductivité hydraulique et la décroissance du taux d'érosion. L'occurrence de ces 2 évolutions représente l'achèvement de la suffusion. Pour la même durée, la masse sèche cumulée représente la réponse de l'échantillon à la sollicitation hydraulique. L'indice de résistance à l'érosion est alors calculé par l'équation (2). Les valeurs obtenues de $I_{\alpha}$ pour les échantillons 5.2-5.7, 6.26.7 et 11.2-11.7 sont respectivement : 4,3, 4,9 et 6,2. En conséquence, les échantillons 5.2-5.7 et 6.2-6.7 sont modérément résistants alors que 1'échantillon 11.2-11.7 est très résistant selon la classification de MAROT et al. (2016).

\subsection{Influence de la suffusion sur la résistance mécanique}

La figure 5(a) présente la contrainte déviatorique en fonction de la déformation axiale pour l'échantillon non érodé 6.2-6.7 et également pour le même sol après l'étape de suffusion. Il importe de noter que le processus de suffusion entraîne dans le cas de cet 


\section{Thème 7 - Risques côtiers}

échantillon, une réduction de la valeur au pic de la contrainte déviatorique de $256 \mathrm{kPa}$ à $208 \mathrm{kPa}$, ce qui correspond à une diminution relative de $19 \%$.

Sur la figure 5(b) sont représentées les valeurs de diminution relative de la contrainte déviatorique au pic en fonction de l'indice de résistance à l'érosion, pour les trois échantillons testés. Ces premiers résultats mettent en évidence la réduction de la résistance mécanique induite par une suffusion complète. Par ailleurs cette diminution est d'autant plus importante que le sol est plus sensible à la suffusion. Toutefois ces résultats étant obtenus pour des sols modérément résistants et très résistants, d'autres essais apparaissent nécessaires pour généraliser la tendance mesurée.

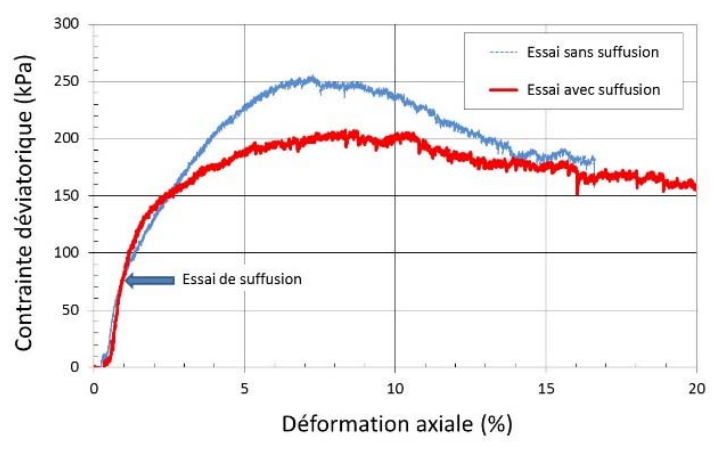

(a)

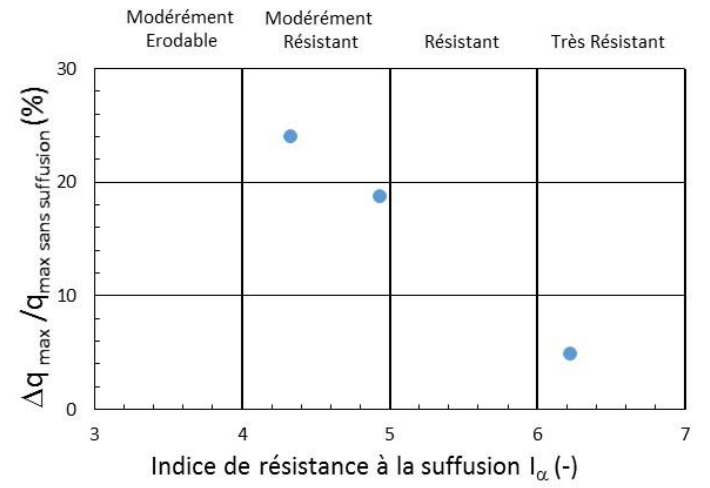

(b)

Figure 5. (a) Contrainte deviatorique en fonction de la déformation axiale (échantillon

6.2-6.7). (b) Diminution relative de contrainte déviatorique au pic en fonction de l'indice de résistance à l'érosion

\section{Conclusion}

Dans cette étude, un nouveau prototype triaxial est utilisé afin de caractériser l'influence de la suffusion sur la résistance mécanique de sols grossiers. Les échantillons testés qui proviennent d'une digue de France sont constitués de sols grossiers de distribution bien graduée. Une série d'essais triaxiaux est réalisée sur des échantillons intacts et des échantillons ayant préalablement subis le processus de suffusion. Cette suffusion a été produite sous gradient hydraulique croissant par palier. La méthode interprétative est basée sur la masse érodée sèche cumulée et l'énergie dissipée par le fluide interstitiel. Cette méthode permet d'établir la sensibilité à la suffusion des échantillons testés à la fin de l'essai de suffusion qui est définie par l'invariabilité de la conductivité hydraulique et la décroissance du taux d'érosion. Dans le cas des sols testés, la suffusion complète induit une diminution de la contrainte déviatorique au pic. 


\section{XVèmes Journées Nationales Génie Côtier - Génie Civil \\ La Rochelle, 29 au 31 mai 2018}

\section{Remerciements}

Les auteurs tiennent à remercier EDF-CIH pour le financement de ces recherches.

\section{Références bibliographiques}

BENDAHMANE F., MAROT D., ALEXIS A. (2008). Experimental parametric Study of Suffusion and Backward Erosion. J. Geotech. Geoenviron. Eng., Vol. 134(1), pp 57-67. https://doi.org/10.1061/(ASCE)1090-0241(2008)134:1(57)

CHANG D.S., ZHANG L.M. (2013a). Extended internal stability criteria for soils under seepage. Soils Found., Vol. 53(4), pp 569-583. https://doi.org/10.1016/j.sandf.2013.06.008 CHANG D.S., ZHANG L.M. (2013b). Critical hydraulic gradients of internal erosion under complex stress states. J. Geotech. Geoenviron. Eng., Vol. 139(9), pp 1454-1467. https://doi.org/10.1061/(ASCE)GT.1943-5606.0000871

FELL R., FRY J.J. (2013). Erosion in geomechanics applied to dams and levees, S. Bonelli Ed., ISTE-Wiley, London, pp 1-99.

KE L., TAKAHASHI A. (2015). Drained monotonic responses of suffusional cohesionless soils. J. Geotech. Geoenviron. Eng., Vol. 41(8). https://doi.org/10.1061/(ASCE)GT.1943-5606.0001327

KENNEY T.C., LAU D. (1985). Internal stability of granular filters. Can. Geotech. J., Vol. 22, pp 215-225. https://doi.org/10.1139/t85-029

MAROT D., REGAZZONI P.L., WAHL T. (2011). Energy based method for providing soil surface erodibility rankings. Journal of Geotechnical and Geoenvironmental Engineering, Vol. 137(12), pp 1290-1294. https://doi.org/10.1061/(ASCE)GT.1943-5606.0000538 MAROT D., ROCHIM A., NGUYEN H.H., BENDAHMANE F., SIBILLE L. (2016). Assessing the susceptibility of gap graded soils to internal erosion: proposition of a new experimental methodology. Natural Hazards, Vol. 83(1), pp 365-388. https://doi.org/10.1007/s11069-016-2319-8

REDDI L.N., LEE I., BONALA M.V.S. (2000). Comparison of internal and surface erosion using flow pump test on a sand-kaolinite mixture. J. ASTM Geotech Test., Vol. 23(1), pp 116-122. https://doi.org/10.1520/GTJ11129J

ROCHIM A., MAROT D., SIBILLE L., LE V.T. (2017). Effect of hydraulic loading history on the characterization of suffusion susceptibility of cohesionless soils. Journal of Geotechnical and Geoenvironmental Engineering, Vol. 143(7). https://doi.org/10.1061/(ASCE)GT.1943-5606.0001673

SCHOLTĖS L., HICHER P.Y., SIBILLE L. (2010). Multiscale approaches to describe mechanical responses induced by particle removal in granular materials. Comptes Rendus Mécanique (CRAS), Vol. 338(10-11), pp 627-638. https://doi.org/10.1016/j.crme.2010.10.003

SKEMPTON A.W., BROGAN J.M. (1994). Experiments on piping in sandy gravels. Géotechnique, Vol. 44(3), pp 440-460. https://doi.org/10.1680/geot.1994.44.3.449 
Thème 7 - Risques côtiers

STERPI D. (2003). Effects of the erosion and transport of fine particles due to seepage flow. International Journal of Geomechanics, Vol. 3(1), pp 111-122. https://doi.org/10.1061/(ASCE)1532-3641(2003)3:1(111)

WAN C.F., FELL R. (2008). Assessing the potential of internal instability and suffusion in embankment dams and their foundations. Journal of Geotechnical and Geoenvironmental Engineering, Vol 134(3), pp 401-407. https://doi.org/10.1061/(ASCE)10900241(2008)134:3(401)

ZHONG C., LE V.T., BENDAHMANE F., MAROT D., ZHENYU Y. (2018). Investigation of spatial scale effects on suffusion susceptibility. Journal of Geotechnical and Geoenvironmental Engineering. Accepté, sous presse. 